\title{
MONOMIAL IDEALS WITH TINY SQUARES
}

\author{
SHALOM ELIAHOU, JÜRGEN HERZOG AND MARYAM MOHAMMADI SAEM
}

\begin{abstract}
Let $I \subset K[x, y]$ be a monomial ideal. How small can $\mu\left(I^{2}\right)$ be in terms of $\mu(I)$ ? It has been expected that the inequality $\mu\left(I^{2}\right)>\mu(I)$ should hold whenever $\mu(I) \geq 2$. Here we disprove this expectation and provide a somewhat surprising answer to the above question.
\end{abstract}

\section{INTRODUCTION}

For an ideal $I$ in a Noetherian ring $R$, let $\mu(I)$ denote as usual the least number of generators of $I$. If $\mu(I)=m$, how small can $\mu\left(I^{2}\right)$ be in terms of $m$ ? Obviously, in suitable rings with zero-divisors, we may have $\mu\left(I^{2}\right)=0$. There even exist onedimensional local domains $(R, \mathfrak{m})$ with the property that the square of their maximal ideal $\mathfrak{m}$ requires less generators than $\mathfrak{m}$ itself, see [1, 2]. However, if $R$ is a regular local ring, or if $R$ is a polynomial ring over a field $K$ and $I$ is a homogeneous ideal of $R$, it has been expected in [3] that the inequality $\mu\left(I^{2}\right)>\mu(I)$ should hold whenever $\mu(I) \geq 2$. This is indeed the case for any integrally closed ideal $I$ in a 2-dimensional regular local ring. On the other hand, it is not too difficult to construct examples of monomial ideals $I$ in a polynomial ring $S$ with at least 4 variables such that $\mu\left(I^{2}\right)<\mu(I)$. However, these examples satisfy height $I<\operatorname{dim} S$. So far no ideals $I$ with $\mu\left(I^{2}\right)<\mu(I)$ were known for 2-dimensional regular rings. In this paper, we shall prove the following statements.

Theorem 1.1. For every integer $m \geq 5$, there exists a monomial ideal $I \subset K[x, y]$ such that $\mu(I)=m$ and $\mu\left(I^{2}\right)=9$.

Moreover, this result is best possible for $m \geq 6$.

Theorem 1.2. Let $I \subset K[x, y]$ be a monomial ideal. If $\mu(I) \geq 6$ then $\mu\left(I^{2}\right) \geq 9$.

Here are some notation to be used throughout. We denote by $\mathcal{M}$ the set of monomials in $K[x, y]$, i.e.

$$
\mathcal{M}=\left\{x^{i} y^{j} \mid i, j \in \mathbb{N}\right\} .
$$

As usual, we view $\mathcal{M}$ as partially ordered by divisibility.

For a monomial ideal $J \subset K[x, y]$, we denote by $\mathcal{G}(J)$ its unique minimal system of monomial generators. It is well known that $\mathcal{G}(J)$ is of cardinality $\mu(J)$ and consists of all monomials in $J$ which are minimal under divisibility, i.e.

$$
\mathcal{G}(J)=(\mathcal{M} \cap J) \backslash(\mathcal{M} \cap J) \mathcal{M}^{*}
$$

where $\mathcal{M}^{*}=\mathcal{M} \backslash\{1\}$. 
Finally, given integers $a \leq b$, we denote by $[a, b]$ the integer interval they span, i.e.

$$
[a, b]=\{c \in \mathbb{Z} \mid a \leq c \leq b\} .
$$

\section{Preliminaries}

Let $m \geq 2$ be an integer and let $I \subset K[x, y]$ be a monomial ideal such that $\mu(I)=m$. Then $\mathcal{G}(I)=\left\{u_{1}, \ldots, u_{m}\right\}$, where $u_{i}=x^{a_{i}} y^{b_{i}}$ for all $i$ and where the exponents $a_{i}, b_{i} \in \mathbb{N}$ satisfy

$$
\begin{gathered}
a_{1}>a_{2}>\cdots>a_{m}, \\
b_{1}<b_{2}<\cdots<b_{m} .
\end{gathered}
$$

Removing any common factor among the $u_{i}$, we may assume $a_{m}=b_{1}=0$ if desired.

Let $V=\left\{(i, j) \in \mathbb{N}^{2} \mid 1 \leq i \leq j \leq m\right\}$, and consider the map

$$
\begin{aligned}
f: \quad V & \rightarrow I^{2} \\
(i, j) & \mapsto u_{i} u_{j} .
\end{aligned}
$$

Then $f(V)$ generates $I^{2}$, but not minimally so in general. Thus $\mathcal{G}\left(I^{2}\right) \subseteq f(V)$, with equality only occurring in special circumstances. How small can $\mathcal{G}\left(I^{2}\right)$ be? So far this was not well understood. We provide a complete answer in this note.

We partially order $\mathbb{N}^{2}$ as follows:

$$
(i, j) \leq(k, l) \Longleftrightarrow i \leq k \text { and } j \leq l \text {. }
$$

Since $V \subset \mathbb{N}^{2}$, we also view $V$ as partially ordered by $\leq$. Recall that $\mathcal{M}$ is partially ordered by divisibility. These orderings interact in a simple yet useful way, as shown by the lemma below. Recall that an antichain in a poset is a subset whose elements are pairwise noncomparable.

Lemma 2.1. Let $v, v^{\prime} \in V$. If $f(v)$ divides $f\left(v^{\prime}\right)$, then either $v=v^{\prime}$ or else $\left\{v, v^{\prime}\right\}$ is an antichain in $V$.

Equivalently, if $v<v^{\prime}$, then $\left\{f(v), f\left(v^{\prime}\right)\right\}$ is an antichain in $\mathcal{M}$.

Proof. Set $v=(i, j), v^{\prime}=(k, l)$, and assume $v<v^{\prime}$. Then $i \leq k$ and $j \leq l$, and at least one of these inequalities is strict. We have $f(v)=u_{i} u_{j}=x^{a_{i}+a_{j}} y^{b_{i}+b_{j}}$. Similarly, $f\left(v^{\prime}\right)=x^{a_{k}+a_{l}} y^{b_{k}+b_{l}}$. Since the $a_{t}$ are decreasing and the $b_{t}$ are increasing, and since either $i<k$ or $j<l$, we have

$$
\begin{aligned}
a_{i}+a_{j} & >a_{k}+b_{l}, \\
b_{i}+b_{j} & <b_{k}+b_{l} .
\end{aligned}
$$

Therefore, neither $u_{i} u_{j}$ divides $u_{k} u_{l}$, nor conversely.

Remark 2.2. If $v, v^{\prime} \in V$, then $v, v^{\prime}$ are noncomparable if and only if $\min v<\min v^{\prime} \leq \max v^{\prime}<\max v$ or $\min v^{\prime}<\min v \leq \max v<\max v^{\prime}$.

Picturing any pair $w=(i, j) \in V$ as an edge joining the vertices $i, j \in \mathbb{N}$, it is useful to keep in mind that graphically, noncomparable pairs $v, v^{\prime} \in V$ look like this: 


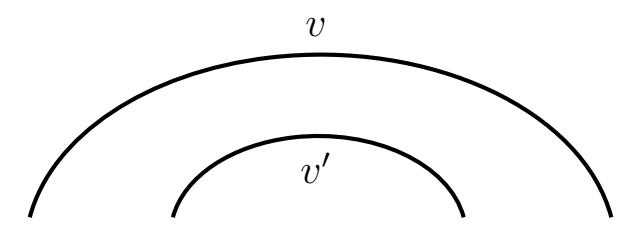

For instance, if $v=(1,3)$, the only element of $V$ which is noncomparable to $v$ is $v^{\prime}=(2,2)$, as is graphically obvious. Therefore by Lemma 2.1, if $u_{1} u_{3} \notin \mathcal{G}\left(I^{2}\right)$, then the only element of $\mathcal{G}\left(I^{2}\right)$ dividing $u_{1} u_{3}$ must be $u_{2}^{2}$.

Of course, noncomparability is not a transitive relation. For instance, the only elements of $V$ which are noncomparable to $v^{\prime}=(2,2)$ are all $v^{\prime \prime} \in V$ of the form $v^{\prime \prime}=(1, j)$ with $j \in[3, m]$. Therefore, if $u_{2}^{2} \notin \mathcal{G}\left(I^{2}\right)$, any minimal generator of $I^{2}$ dividing it must be of the form $u_{1} u_{j}$ for some a priori unspecified $j \in[3, m]$.

We shall also need the next lemma.

Lemma 2.3. Let $v, v_{1}, v_{2} \in V$. Assume that $v_{1} \leq v_{2}$ and that $f(v)$ divides both $f\left(v_{1}\right), f\left(v_{2}\right)$. Then $f(v)$ divides $f\left(v^{\prime}\right)$ for all $v^{\prime} \in V$ such that $v_{1} \leq v^{\prime} \leq v_{2}$.

Proof. Set $v=(i, j), v_{1}=\left(i_{1}, j_{1}\right), v_{2}=\left(i_{2}, j_{2}\right), v^{\prime}=(r, s)$. The hypotheses, together with the respective monotonicity of the $a_{t}$ and $b_{t}$, imply

$$
\begin{aligned}
a_{i}+a_{j} & \leq a_{i_{2}}+a_{j_{2}}, \\
b_{i}+b_{j} & \leq b_{i_{1}}+b_{j_{1}} .
\end{aligned}
$$

Since $i_{1} \leq r \leq i_{2}$ and $j_{1} \leq s \leq j_{2}$, it follows that

$$
\begin{aligned}
a_{i_{2}}+a_{j_{2}} & \leq a_{r}+a_{s}, \\
b_{i_{1}}+b_{j_{1}} & \leq b_{r}+b_{s} .
\end{aligned}
$$

Thus $a_{i}+a_{j} \leq a_{r}+a_{s}$ and $b_{i}+b_{j} \leq b_{r}+b_{s}$, whence $f(v)$ divides $f\left(v^{\prime}\right)$.

\section{CONDITIONS FOR TINY SQUARES}

We now give conditions on a monomial ideal $I=\left(u_{1}, \ldots, u_{m}\right)$ in $K[x, y]$ which will force $\mu\left(I^{2}\right)$ to be a small constant.

Theorem 3.1. Let $m \geq 5$. Let $I=\left(u_{1}, \ldots, u_{m}\right) \subset K[x, y]$ be a monomial ideal with $u_{i}=x^{a_{i}} y^{b_{i}}$ for all $i$, where $a_{1}>\cdots>a_{m}$ and $b_{1}<\cdots<b_{m}$. Assume that the following divisibility conditions hold:

$$
\begin{array}{r|l}
u_{1} u_{m} & u_{2} u_{m-1} \\
u_{1} u_{m-1} & u_{2} u_{3}, u_{m-2}^{2} \\
u_{2}^{2} & u_{1} u_{3}, u_{1} u_{m-2} \\
u_{2} u_{m} & u_{3} u_{m-1}, u_{m-2} u_{m-1} \\
u_{m-1}^{2} & u_{3} u_{m}, u_{m-2} u_{m} .
\end{array}
$$

Then $\mu\left(I^{2}\right)=9$. More precisely, $I^{2}$ is minimally generated by the set

$$
G=\left\{u_{1}^{2}, u_{1} u_{2}, u_{2}^{2}\right\} \cup\left\{u_{1} u_{m-1}, u_{1} u_{m}, u_{2} u_{m}\right\} \cup\left\{u_{m-1}^{2}, u_{m-1} u_{m}, u_{m}^{2}\right\} .
$$


Proof. To see that $G$ generates $I^{2}$, we must show that, for all $i, j \in[1, m]$ such that $i \leq j$, the monomial $u_{i} u_{j}$ is a multiple of some element in $G$. We distinguish several cases.

Case $i=1$. If $j \in\{1,2, m-1, m\}$, we are done since the corresponding monomial $u_{1} u_{j}$ belongs to $G$. Assume now $j \in[3, m-2]$. Then $(1,3) \leq(1, j) \leq(1, m-2)$, and since $u_{2}^{2}$ divides both $u_{1} u_{3}$ and $u_{1} u_{m-2}$ by (4), it also divides $u_{1} u_{j}$ by Lemma 2.3 .

Case $i, j \in[2, m-2]$. If $(i, j)=(2,2)$, we are done since $u_{2}^{2} \in G$. If $(i, j) \neq(2,2)$, then $(2,3) \leq(i, j) \leq(m-2, m-2)$, and since $u_{1} u_{m-1}$ divides both $u_{2} u_{3}$ and $u_{m-2}^{2}$ by (3), it follows from Lemma 2.3 that $u_{1} u_{m-1}$ also divides $u_{i} u_{j}$.

Case $j=m-1$. If $i=2$, then by (21), $u_{2} u_{m-1}$ is divisible by $u_{1} u_{m}$ and $u_{1} u_{m} \in G$. If $i \in[3, m-2]$, then $(3, m-1) \leq(i, m-1) \leq(m-2, m-1)$, and since $u_{2} u_{m}$ divides both $u_{3} u_{m-1}$ and $u_{m-2} u_{m-1}$ by (15), it also divides $u_{i} u_{m-1}$ by Lemma 2.3 . Finally, if $i=m-1$, we are done since $u_{m-1}^{2} \in G$.

Case $j=m$. If $i=2$, we are done since $u_{2} u_{m} \in G$. If $i \in[3, m-2]$, then $(3, m) \leq(i, m) \leq(m-2, m)$, and since $u_{m-1}^{2}$ divides both $u_{3} u_{m}$ and $u_{m-2} u_{m}$ by (6) , it also divides $u_{i} u_{m}$ by Lemma 2.3. Finally, if $i \in\{m-1, m\}$, we are done since $u_{m-1} u_{m}, u_{m}^{2} \in G$.

We conclude that $G$ generates $I^{2}$. To establish the equality $G=\mathcal{G}\left(I^{2}\right)$, it remains to see that $G$ is an antichain. Let $A \subset V$ consist of nine pairs $(i, j)$ such that $G=\left\{u_{i} u_{j} \mid(i, j) \in A\right\}$, for instance

$A=\{(1,1),(1,2),(2,2),(1, m-1),(1, m),(2, m),(m-1, m-1),(m-1, m),(m, m)\}$.

Replacing $(2,2)$ by $(1,3)$ and $(m-1, m-1)$ by $(m-2, m)$ yields a chain in $V$, namely

$$
\begin{aligned}
& (1,1)<(1,2)<(1,3)< \\
& (1, m-1)<(1, m)<(2, m)< \\
& (m-2, m)<(m-1, m)<(m, m) .
\end{aligned}
$$

It follows from Lemma 2.1 that the set

$$
G^{\prime}=G \cup\left\{u_{1} u_{3}, u_{m-2} u_{m}\right\} \backslash\left\{u_{2}^{2}, u_{m-1}^{2}\right\}
$$

is an antichain. In particular, its subset $G \backslash\left\{u_{2}^{2}, u_{m-1}^{2}\right\}$ also is. Consider now $u_{1} u_{3}$. Since $G$ generates $I^{2}$, then $u_{1} u_{3}$ is a multiple of some element of $G$. Moreover, since $G^{\prime}$ is an antichain, the only possible factors of $u_{1} u_{3}$ in $G$ are $u_{2}^{2}$ and $u_{m-1}^{2}$. But since $(1,3)<(m-1, m-1)$, Lemma 2.1 implies that $\left\{u_{1} u_{3}, u_{m-1}^{2}\right\}$ is an antichain. Therefore, the only factor of $u_{1} u_{3}$ in $G$ is $u_{2}^{2}$. Hence, since any monomial in $I^{2}$ has at least one factor in $G \cap \mathcal{G}\left(I^{2}\right)$, it follows that $u_{2}^{2} \in \mathcal{G}\left(I^{2}\right)$.

Let us now see that $u_{2}^{2}$ cannot divide another member of $G$. By Lemma 2.1, the only possible multiples of $u_{2}^{2}$ in $G$ would be $u_{1} u_{m-1}$ and $u_{1} u_{m}$. But neither possibility arises, since $u_{1} u_{m-1}$ divides $u_{2} u_{3}$ by (3) , and $u_{1} u_{m}$ divides $u_{2} u_{m-1}$ by (2).

Entirely symmetric arguments apply to $u_{m-1}^{2}$. We conclude that $G=\mathcal{G}\left(I^{2}\right)$, as desired. 


\section{An explicit CONSTRUCTION}

We now show that the conditions of Theorem 3.1 ensuring $\mu\left(I^{2}\right)=9$ are realizable.

Proposition 4.1. Let $m \geq 5$. Let $I=\left(u_{1}, \ldots, u_{m}\right)$, where $u_{i}=x^{a_{i}} y^{b_{i}}$ with exponents $a_{i}, b_{i}$ defined as follows:

$$
\begin{aligned}
\left(a_{1}, \ldots, a_{m}\right) & =(5 m, 4 m, 4 m-1, \ldots, 3 m+4, m, 0), \\
\left(b_{1}, \ldots, b_{m}\right) & =\left(a_{m}, \ldots, a_{1}\right) .
\end{aligned}
$$

Then $\mu(I)=m$ and $\mu\left(I^{2}\right)=9$.

Proof. Since the $a_{i}$ are decreasing and the $b_{i}$ are increasing, the $u_{i}$ constitute an antichain and hence a minimal system of generators of $I$. In order to show $\mu\left(I^{2}\right)=9$, it suffices to prove that the divisibility conditions of Theorem 3.1 are met. This is straightforward. For convenience, here are the monomials involved:

$$
\begin{array}{lll}
u_{1} u_{m}=x^{5 m} y^{5 m} & u_{2} u_{m-1}=x^{5 m} y^{5 m} & \\
u_{1} u_{m-1}=x^{6 m} y^{4 m} & u_{2} u_{3}=x^{8 m-1} y^{4 m+4} & u_{m-2}^{2}=x^{6 m+8} y^{8 m-2} \\
u_{2}^{2}=x^{8 m} y^{2 m} & u_{1} u_{3}=x^{9 m-1} y^{3 m+4} & u_{1} u_{m-2}=x^{8 m+4} y^{4 m-1} \\
u_{2} u_{m}=x^{4 m} y^{6 m} & u_{3} u_{m-1}=x^{5 m-1} y^{7 m+4} & u_{m-2} u_{m-1}=x^{4 m+4} y^{8 m-1} \\
u_{m-1}^{2}=x^{2 m} y^{8 m} & u_{3} u_{m}=x^{4 m-1} y^{8 m+4} & u_{m-2} u_{m}=x^{3 m+4} y^{9 m-1}
\end{array}
$$

Let us now look at the degree distribution of $\mathcal{G}(I)$ and of $\mathcal{G}\left(I^{2}\right)$. The generators $u_{i}$ of $I$ are of degree $a_{i}+b_{i}=a_{i}+a_{m+1-i}$ for all $i \in[1, m]$. For $m \geq 5$, we find

$$
\begin{aligned}
\operatorname{deg}\left(u_{1}\right)=\operatorname{deg}\left(u_{2}\right)=\operatorname{deg}\left(u_{m-1}\right)=\operatorname{deg}\left(u_{m}\right) & =5 m, \\
\operatorname{deg}\left(u_{i}\right)=\operatorname{deg}\left(u_{m+1-i}\right) & =7 m+3
\end{aligned}
$$

for all $3 \leq i \leq m-2$. That is, the ideal $I$ is generated in two degrees only. For $I^{2}$ the situation is even simpler. It is generated in the single degree $10 \mathrm{~m}$, the common degree of its nine minimal generators.

Example 4.2. Here is the case $m=10$ of Proposition 4.1. We have

$$
I=\left(x^{50}, x^{40} y^{10}, x^{39} y^{34}, x^{38} y^{35}, x^{37} y^{36}, x^{36} y^{37}, x^{35} y^{38}, x^{34} y^{39}, x^{10} y^{40}, y^{50}\right)
$$

and

$$
I^{2}=\left(x^{100}, x^{90} y^{10}, x^{80} y^{20}, x^{60} y^{40}, x^{50} y^{50}, x^{40} y^{60}, x^{20} y^{80}, x^{10} y^{90}, y^{100}\right) .
$$

To conclude this section, let us show that in contrast, if $I$ is generated in a single degree, then $\mu\left(I^{2}\right)$ grows to infinity with $\mu(I)$.

Proposition 4.3. Let $I \subset K[x, y]$ be a monomial ideal generated in a single degree. If $\mu(I)=m$ then $\mu\left(I^{2}\right) \geq 2 m-1$.

Proof. Set $\mathcal{G}(I)=\left\{u_{1}, \ldots, u_{m}\right\}$, and assume $\operatorname{deg}\left(u_{i}\right)=d$ for all $i$. Since $\operatorname{deg}\left(u_{i} u_{j}\right)=$ $2 d$ for all $i, j$, the distinct $u_{i} u_{j}$ form an antichain for divisibility. Hence $\mu\left(I^{2}\right)$ equals the number of pairwise distinct $u_{i} u_{j}$. Now that number is at least $2 m-1$, as witnessed by the subset

$$
u_{1} u_{1}, u_{1} u_{2}, \ldots, u_{1} u_{m}, u_{2} u_{m}, \ldots, u_{m} u_{m}
$$




\section{Computing $\mu\left(I^{k}\right)$}

For the ideal $I$ satisfying $\mu(I)=m, \mu\left(I^{2}\right)=9$ of the preceding section, we now determine $\mu\left(I^{k}\right)$ for all $k$.

Proposition 5.1. Given $m \geq 5$, let $I=\left(u_{1}, \ldots, u_{m}\right)$ be the ideal defined in Proposition 4.1. For all $k \geq 3$, we have $\mu\left(I^{k}\right)=5 k+1$.

Proof. It follows from Theorem 3.1 that $I^{2}$ is minimally generated by

$$
G=\left\{u_{1}^{2}, u_{1} u_{2}, u_{2}^{2}\right\} \cup\left\{u_{1} u_{m-1}, u_{1} u_{m}, u_{2} u_{m}\right\} \cup\left\{u_{m-1}^{2}, u_{m-1} u_{m}, u_{m}^{2}\right\} .
$$

Let $J=\left(u_{1}, u_{2}, u_{m-1}, u_{m}\right)$ and $H=\left(u_{3}, \ldots, u_{m-2}\right)$, so that $I=J+H$. Then $I^{2}=J^{2}$, since $I^{2}=(G) \subseteq J^{2} \subseteq I^{2}$. Hence $I J, I H \subseteq J^{2}$, from which it follows that $I^{k}=J^{k}$ for all $k \geq 3$.

Now, by construction in Proposition 4.1, we have $J=\left(x^{5 m}, x^{4 m} y^{m}, x^{m} y^{4 m}, y^{5 m}\right)$. Let $J_{0}=\left(x^{5}, x^{4} y, x y^{4}, y^{5}\right)$. Then $\mu\left(J^{k}\right)=\mu\left(J_{0}^{k}\right)$ for all $k \geq 1$. Moreover, we have

$$
J_{0}^{3}=\left(x^{15}, x^{14} y, \ldots, x y^{14}, y^{15}\right)
$$

as easily seen. It follows that $J_{0}^{k}=(x, y)^{5 k}$ for all $k \geq 3$. Therefore

$$
\mu\left(I^{k}\right)=\mu\left(J^{k}\right)=\mu\left(J_{0}^{k}\right)=5 k+1
$$

for all $k \geq 3$, as stated.

Corollary 5.2. For any integer $k_{0} \geq 2$, there exists a monomial ideal $I$ in $K[x, y]$ such that $\mu\left(I^{k}\right)<\mu(I)$ for all $k$ such that $2 \leq k \leq k_{0}$.

Proof. Let $m>5 k_{0}+1$. By Propositions 4.1 and 5.1, there is an ideal $I$ with $\mu(I)=m>5 k_{0}+1$ and satisfying $\mu\left(I^{k}\right) \leq 5 k+1$ for all $k \geq 2$. Therefore $\mu\left(I^{k}\right)<\mu(I)$ for all $2 \leq k \leq k_{0}$.

\section{Optimality}

We now prove that Theorem 3.1 is best possible, in the sense that $\mu\left(I^{2}\right)$ is bounded below by 9 if $\mu(I) \geq 6$. For that we need some more notation. If $I \subset K[x, y]$ is a monomial ideal with $\mathcal{G}(I)=\left\{u_{1}, \ldots, u_{m}\right\}$, let us denote

$$
\mathcal{G}^{2}(I)=\left\{u_{i} u_{j} \mid 1 \leq i \leq j \leq m\right\},
$$

i.e. $\mathcal{G}^{2}(I)=f(V)$ in the notation of the Introduction. Let

$$
\gamma: \mathcal{G}^{2}(I) \rightarrow \mathcal{G}\left(I^{2}\right)
$$

be the map defined, for all $u_{i} u_{j} \in \mathcal{G}^{2}(I)$, by

$$
\gamma\left(u_{i} u_{j}\right)=u_{k} u_{l}
$$

where $u_{k} u_{l} \in \mathcal{G}\left(I^{2}\right)$ is the lexicographically first minimal monomial generator of $I^{2}$ dividing $u_{i} u_{j}$. Note that $\gamma \circ \gamma=\gamma$. Finally, given $u_{i} u_{j} \in \mathcal{G}^{2}(I)$, we denote

$$
\operatorname{div}\left(u_{i} u_{j}\right)=\left\{u_{r} u_{s} \in \mathcal{G}^{2}(I) \mid u_{r} u_{s} \text { divides } u_{i} u_{j}\right\} .
$$

Of course $\gamma\left(u_{i} u_{j}\right) \in \operatorname{div}\left(u_{i} u_{j}\right)$. We will repeatedly use the following reformulation of Lemma 2.1. 
Lemma 6.1. For all $(i, j) \in V$, we have

$$
\operatorname{div}\left(u_{i} u_{j}\right) \subseteq\left\{u_{i} u_{j}\right\} \cup\left\{u_{r} u_{s} \in \mathcal{G}^{2}(I) \mid(r, s) \text { is noncomparable to }(i, j) \text { in } V\right\} .
$$

Proof. As stated in Lemma2.1, if $u_{r} u_{s}$ divides $u_{i} u_{j}$ and $(r, s) \neq(i, j)$, then $\{(r, s),(i, j)\}$ is an antichain in $V$.

Thus for instance, $\operatorname{div}\left(u_{1} u_{3}\right) \subseteq\left\{u_{1} u_{3}, u_{2}^{2}\right\}$ as already observed after Remark 2.2. Again, it is useful to keep in mind the picture representing noncomparable pairs following that remark.

Here is the main result of this section.

Theorem 6.2. Let $I \subset K[x, y]$ be a monomial ideal. If $\mu(I) \geq 6$ then $\mu\left(I^{2}\right) \geq 9$.

Proof. Let $m=\mu(I)$. As before, we denote $\mathcal{G}(I)=\left\{u_{1}, \ldots, u_{m}\right\}$, where $u_{i}=x^{a_{i}} y^{b_{i}}$ for all $i$ and where the $a_{i}, b_{i}$ are decreasing and increasing, respectively. We start by considering the following six monomials in $\mathcal{G}^{2}(I)$ :

$$
u_{1}^{2}, u_{1} u_{2}, u_{1} u_{3}, u_{m-2} u_{m}, u_{m-1} u_{m}, u_{m}^{2} .
$$

By Lemma 6.1, we have $\gamma\left(u_{1}^{2}\right)=u_{1}^{2}$ since $(1,1)$ is comparable to every $(i, j) \in V$, namely $(1,1) \leq(i, j)$. Similarly, each one of the pairs $(1,2),(m-1, m),(m, m)$ is comparable to all elements of $V$, whence

$$
\gamma\left(u_{1} u_{2}\right)=u_{1} u_{2}, \gamma\left(u_{m-1} u_{m}\right)=u_{m-1} u_{m}, \gamma\left(u_{m}^{2}\right)=u_{m}^{2}
$$

Consider $u_{1} u_{3}$. Then

$$
\gamma\left(u_{1} u_{3}\right) \in \operatorname{div}\left(u_{1} u_{3}\right) \subseteq\left\{u_{1} u_{3}, u_{2}^{2}\right\}
$$

by Lemma 6.1, since $(1,3)$ is comparable to all elements of $V$ except $(2,2)$. Symmetrically, we have

$$
\gamma\left(u_{m-2} u_{m}\right) \in\left\{u_{m-2} u_{m}, u_{m-1}^{2}\right\} .
$$

Thus so far, we have six pairwise distinct minimal monomial generators of $I^{2}$, namely

$$
A=\left\{u_{1}^{2}, u_{1} u_{2}, \gamma\left(u_{1} u_{3}\right), \gamma\left(u_{m-2} u_{m}\right), u_{m-1} u_{m}, u_{m}^{2}\right\} \subseteq \mathcal{G}\left(I^{2}\right) .
$$

Considering $u_{1} u_{m}$, we distinguish two cases.

Case 1: $\gamma\left(u_{1} u_{m}\right) \neq u_{1} u_{m}$. Therefore $u_{1} u_{m} \notin \mathcal{G}\left(I^{2}\right)$, and $\gamma\left(u_{1} u_{m}\right)=u_{r} u_{s}$ for some $r, s \in[2, m-1]$ such that $r \leq s$. Since $\operatorname{div}\left(u_{2} u_{3}\right) \cap \operatorname{div}\left(u_{m-2} u_{m-1}\right) \subseteq\left\{u_{1} u_{m}\right\}$ by Lemma 6.1, it follows that $\gamma\left(u_{2} u_{3}\right) \neq \gamma\left(u_{m-2} u_{m-1}\right)$. Moreover, it also follows from Lemma 6.1 that $\gamma\left(u_{2} u_{3}\right), \gamma\left(u_{m-2} u_{m-1}\right) \notin A$. So let

$$
A^{\prime}=A \sqcup\left\{\gamma\left(u_{2} u_{3}\right), \gamma\left(u_{m-2} u_{m-1}\right)\right\} .
$$

Then $\operatorname{card}\left(A^{\prime}\right)=8$. There are two subcases.

Case (1.1) If $\gamma\left(u_{1} u_{m}\right) \notin A^{\prime}$, then we are done, since joining $\gamma\left(u_{1} u_{m}\right)$ to $A^{\prime}$ yields nine minimal generators of $I^{2}$.

Case (1.2) If $\gamma\left(u_{1} u_{m}\right) \in A^{\prime}$, then by Lemma 6.1, the only possibilities are as follows:

$$
\begin{array}{ll}
\gamma\left(u_{1} u_{m}\right)=u_{2}^{2} & =\gamma\left(u_{1} u_{3}\right), \\
\gamma\left(u_{1} u_{m}\right)=u_{2} u_{3} & =\gamma\left(u_{2} u_{3}\right), \\
\gamma\left(u_{1} u_{m}\right)=u_{m-2} u_{m-1} & =\gamma\left(u_{m-2} u_{m-1}\right) \\
\gamma\left(u_{1} u_{m}\right)=u_{m-1}^{2} & =\gamma\left(u_{m-1}^{2}\right)
\end{array}
$$


But then in each case, Lemma 6.1 implies $\gamma\left(u_{2} u_{m-2}\right) \notin A^{\prime}$, and we are done again.

Case 2: $\gamma\left(u_{1} u_{m}\right)=u_{1} u_{m}$. Then $u_{1} u_{m} \in \mathcal{G}\left(I^{2}\right)$. Let

$$
A^{\prime}=A \sqcup\left\{u_{1} u_{m}\right\}=\left\{u_{1}^{2}, u_{1} u_{2}, \gamma\left(u_{1} u_{3}\right), \gamma\left(u_{m-2} u_{m}\right), u_{m-1} u_{m}, u_{m}^{2}, u_{1} u_{m}\right\} .
$$

Then $A^{\prime} \subseteq \mathcal{G}\left(I^{2}\right)$ and $\operatorname{card}\left(A^{\prime}\right)=7$. Comparing $\gamma\left(u_{1} u_{m-1}\right)$ to $\gamma\left(u_{1} u_{3}\right)$, and $\gamma\left(u_{2} u_{m}\right)$ to $\gamma\left(u_{m-2} u_{m}\right)$, gives rise to four subcases. Let us consider them in turn.

Case (2.1): $\gamma\left(u_{1} u_{m-1}\right)=\gamma\left(u_{1} u_{3}\right), \gamma\left(u_{2} u_{m}\right)=\gamma\left(u_{m-2} u_{m}\right)$.

Since $\operatorname{div}\left(u_{1} u_{m-1}\right) \cap \operatorname{div}\left(u_{1} u_{3}\right) \subseteq\left\{u_{2}^{2}\right\}$, we have $\gamma\left(u_{1} u_{m-1}\right)=\gamma\left(u_{1} u_{3}\right)=u_{2}^{2}$. Similarly, we have $\gamma\left(u_{2} u_{m}\right)=\gamma\left(u_{m-2} u_{m}\right)=u_{m-1}^{2}$. Therefore

$$
\begin{array}{r|l}
u_{2}^{2} & u_{1} u_{m-1}, \\
u_{m-1}^{2} & u_{2} u_{m} .
\end{array}
$$

Hence $u_{2}^{2} u_{m-1}^{2} \mid u_{1} u_{m-1} u_{2} u_{m}$, implying $u_{2} u_{m-1} \mid u_{1} u_{m}$. Whence

$$
u_{2} u_{m-1}=u_{1} u_{m}
$$

since $u_{1} u_{m} \in \mathcal{G}\left(I^{2}\right)$. Let

$$
A^{\prime \prime}=A^{\prime} \cup\left\{\gamma\left(u_{2} u_{3}\right), \gamma\left(u_{m-2} u_{m-1}\right)\right\} .
$$

Then $A^{\prime \prime} \subseteq \mathcal{G}\left(I^{2}\right)$ by construction. We claim that card $\left(A^{\prime \prime}\right)=9$. Indeed, Lemma 6.1 implies

$$
\left\{\gamma\left(u_{2} u_{3}\right), \gamma\left(u_{m-2} u_{m-1}\right)\right\} \cap A^{\prime} \subseteq\left\{u_{1} u_{m}\right\} .
$$

But since $u_{1} u_{m}=u_{2} u_{m-1}$ here, as observed in (9), it follows that

$$
u_{1} u_{m} \notin \operatorname{div}\left(u_{2} u_{3}\right) \cup \operatorname{div}\left(u_{m-2} u_{m-1}\right),
$$

whence the above intersection is empty. Moreover, since $\operatorname{div}\left(u_{2} u_{3}\right) \cap \operatorname{div}\left(u_{m-2} u_{m-1}\right) \subseteq$ $\left\{u_{1} u_{m}\right\}$, it follows from (10) that $\gamma\left(u_{2} u_{3}\right) \neq \gamma\left(u_{m-2} u_{m-1}\right)$. Therefore $\operatorname{card}\left(A^{\prime \prime}\right)=9$ as claimed, and we are done in this case.

Case (2.2): $\gamma\left(u_{1} u_{m-1}\right)=\gamma\left(u_{1} u_{3}\right), \gamma\left(u_{2} u_{m}\right) \neq \gamma\left(u_{m-2} u_{m}\right)$.

This is by far the most delicate case. As observed in the preceding case, the equality $\gamma\left(u_{1} u_{m-1}\right)=\gamma\left(u_{1} u_{3}\right)$ implies $\gamma\left(u_{1} u_{m-1}\right)=\gamma\left(u_{1} u_{3}\right)=u_{2}^{2}$. Now $\gamma\left(u_{2} u_{m}\right) \notin$ $A^{\prime}$, as follows from Lemma 6.1 and the inequality $\gamma\left(u_{2} u_{m}\right) \neq \gamma\left(u_{m-2} u_{m}\right)$. Let

$$
A^{\prime \prime}=A^{\prime} \cup\left\{\gamma\left(u_{2} u_{m}\right)\right\} \text {. }
$$

Then $A^{\prime \prime} \subseteq \mathcal{G}\left(I^{2}\right)$ and $\operatorname{card}\left(A^{\prime \prime}\right)=8$. We distinguish four subcases, according to the values of $\gamma\left(u_{2} u_{m}\right)$ and $\gamma\left(u_{m-2} u_{m}\right)$. Recall that $\gamma\left(u_{m-2} u_{m}\right) \in\left\{u_{m-2} u_{m}, u_{m-1}^{2}\right\}$.

Case (2.2.1): $\gamma\left(u_{2} u_{m}\right)=u_{2} u_{m}, \gamma\left(u_{m-2} u_{m}\right)=u_{m-2} u_{m}$.

Then

$$
A^{\prime \prime}=\left\{u_{1}^{2}, u_{1} u_{2}, u_{2}^{2}, u_{m-2} u_{m}, u_{m-1} u_{m}, u_{m}^{2}, u_{1} u_{m}, u_{2} u_{m}\right\} .
$$

Interestingly here, for $m=5$, there are explicit cases where $A^{\prime \prime}=\mathcal{G}\left(I^{2}\right)$. Now under our hypothesis $m \geq 6$, a ninth element in $\mathcal{G}\left(I^{2}\right) \backslash A^{\prime \prime}$ is given by $\gamma\left(u_{3} u_{m}\right)$, as easily follows from the present shape of $A^{\prime \prime}$ and Lemma 6.1.

Case (2.2.2): $\gamma\left(u_{2} u_{m}\right)=u_{2} u_{m}, \gamma\left(u_{m-2} u_{m}\right)=u_{m-1}^{2}$.

Then

$$
A^{\prime \prime}=\left\{u_{1}^{2}, u_{1} u_{2}, u_{2}^{2}, u_{m-1}^{2}, u_{m-1} u_{m}, u_{m}^{2}, u_{1} u_{m}, u_{2} u_{m}\right\} .
$$


Consider $u_{2} u_{m-1}$. If $\gamma\left(u_{2} u_{m-1}\right) \notin A^{\prime \prime}$, we have our ninth minimal generator and we are done. Assume now for a contradiction that $\gamma\left(u_{2} u_{m-1}\right) \in A^{\prime \prime}$. Then under the present shape of $A^{\prime \prime}$ and Lemma 6.1, the only possibility is $\gamma\left(u_{2} u_{m-1}\right)=u_{1} u_{m}$. Therefore

$$
\begin{array}{r|l}
u_{2}^{2} & u_{1} u_{m-1}, \\
u_{1} u_{m} & u_{2} u_{m-1} .
\end{array}
$$

Hence $u_{1} u_{2}^{2} u_{m} \mid u_{1} u_{2} u_{m-1}^{2}$, implying $u_{2} u_{m} \mid u_{m-1}^{2}$. This is a contradiction, since $u_{m-1}^{2}=$ $\gamma\left(u_{m-2} u_{m}\right)$, whence $u_{m-1}^{2}$ divides $u_{m-2} u_{m}$ which is not divisible by $u_{2} u_{m}$.

Case (2.2.3): $\gamma\left(u_{2} u_{m}\right) \neq u_{2} u_{m}, \gamma\left(u_{m-2} u_{m}\right)=u_{m-2} u_{m}$.

By Lemma 6.1, we have $\gamma\left(u_{2} u_{m}\right)=u_{r} u_{s}$ for some $3 \leq r \leq s \leq m-1$. Then

$$
A^{\prime \prime}=\left\{u_{1}^{2}, u_{1} u_{2}, u_{2}^{2}, u_{m-2} u_{m}, u_{m-1} u_{m}, u_{m}^{2}, u_{1} u_{m}, u_{r} u_{s}\right\} .
$$

Consider $u_{m-3} u_{m}$. If $\gamma\left(u_{m-3} u_{m}\right) \notin A^{\prime \prime}$, we have our ninth minimal generator and we are done. If $\gamma\left(u_{m-3} u_{m}\right) \in A^{\prime \prime}$, then the only possibility allowed by Lemma 6.1 is $\gamma\left(u_{m-3} u_{m}\right)=u_{r} u_{s}$. The same lemma then implies $m-2 \leq r \leq s \leq m-1$.

Consider now $u_{2} u_{3}$. If $\gamma\left(u_{2} u_{3}\right) \notin A^{\prime \prime}$, we are done. Assume now for a contradiction that $\gamma\left(u_{2} u_{3}\right) \in A^{\prime \prime}$. Then the only possibility is $\gamma\left(u_{2} u_{3}\right)=u_{1} u_{m}$. Therefore, since $\gamma\left(u_{1} u_{3}\right)=u_{2}^{2}$ in the present case, we have

$$
\begin{array}{r|l}
u_{2}^{2} & u_{1} u_{3}, \\
u_{1} u_{m} & u_{2} u_{3} .
\end{array}
$$

Hence $u_{1} u_{2}^{2} u_{m} \mid u_{1} u_{2} u_{3}^{2}$, implying $u_{2} u_{m} \mid u_{3}^{2}$. This is a contradiction, since $\gamma\left(u_{2} u_{m}\right)=$ $u_{r} u_{s}$, whence $u_{r} u_{s}$ divides $u_{2} u_{m}$ but cannot divide $u_{3}^{2}$ since $r, s \in[m-2, m-1]$.

Case (2.2.4): $\gamma\left(u_{2} u_{m}\right) \neq u_{2} u_{m}, \gamma\left(u_{m-2} u_{m}\right)=u_{m-1}^{2}$.

As above, we have $\gamma\left(u_{2} u_{m}\right)=u_{r} u_{s}$ for some $3 \leq r \leq s \leq m-1$, and here with $(r, s) \neq(m-1, m-1)$ since $\gamma\left(u_{2} u_{m}\right) \neq \gamma\left(u_{m-2} u_{m}\right)$ in the present Case (2.2). Then

$$
A^{\prime \prime}=\left\{u_{1}^{2}, u_{1} u_{2}, u_{2}^{2}, u_{m-1}^{2}, u_{m-1} u_{m}, u_{m}^{2}, u_{1} u_{m}, u_{r} u_{s}\right\} .
$$

To settle this case, it suffices to show that either $\gamma\left(u_{2} u_{m-1}\right)$ or $\gamma\left(u_{m-2} u_{m-1}\right)$ lies outside $A^{\prime \prime}$. Assume for a contradiction the contrary, i.e.

$$
\left\{\gamma\left(u_{2} u_{m-1}\right), \gamma\left(u_{m-2} u_{m-1}\right)\right\} \subset A^{\prime \prime} .
$$

Lemma 6.1 then implies

$$
\left\{\gamma\left(u_{2} u_{m-1}\right), \gamma\left(u_{m-2} u_{m-1}\right)\right\} \subseteq\left\{u_{1} u_{m}, u_{r} u_{s}\right\} .
$$

On the other hand, that same lemma implies

$$
\operatorname{div}\left(u_{2} u_{m-1}\right) \cap \operatorname{div}\left(u_{m-2} u_{m-1}\right) \subseteq\left\{u_{1} u_{m}\right\} .
$$

We cannot have $\gamma\left(u_{m-2} u_{m-1}\right)=u_{1} u_{m}$. For otherwise, in the present Case (2.2.4), that equality would imply

$$
\begin{array}{l|l}
u_{1} u_{m} & u_{m-2} u_{m-1}, \\
u_{m-1}^{2} & u_{m-2} u_{m} . \\
& 9
\end{array}
$$


Therefore $u_{1} u_{m-1}^{2} u_{m} \mid u_{m-2}^{2} u_{m-1} u_{m}$, whence $u_{1} u_{m-1} \mid u_{m-2}^{2}$. This is impossible, since $u_{2}^{2}$ divides $u_{1} u_{m-1}$ in the present Case (2.2), whereas $u_{2}^{2}$ cannot divide $u_{m-2}^{2}$ by Lemma 6.1.

Therefore $\gamma\left(u_{m-2} u_{m-1}\right) \neq u_{1} u_{m}$, whence $\gamma\left(u_{m-2} u_{m-1}\right)=u_{r} u_{s}$ by (12). Since $r, s \in[3, m-1]$, this and Lemma 6.1 imply $(r, s)=(m-2, m-1)$. That is, we have

$$
\gamma\left(u_{m-2} u_{m-1}\right)=u_{r} u_{s}=u_{m-2} u_{m-1} .
$$

Consider now $u_{2} u_{m-1}$. It follows from (12), (13) and (14), that $\gamma\left(u_{2} u_{m-1}\right)=u_{1} u_{m}$. In the present Case (2.2), this yields

$$
\begin{array}{r|l}
u_{1} u_{m} & u_{2} u_{m-1}, \\
u_{2}^{2} & u_{1} u_{m-1}
\end{array}
$$

Therefore $u_{2} u_{m} \mid u_{m-1}^{2}$. But $u_{m-1}^{2}$ divides $u_{m-2} u_{m}$ in the present Case (2.2.4), yet $u_{2} u_{m}$ cannot divide $u_{m-2} u_{m}$. This contradiction shows that (11) is absurd. Therefore

$$
\operatorname{card}\left(A^{\prime \prime} \cup\left\{\gamma\left(u_{2} u_{m-1}\right), \gamma\left(u_{m-2} u_{m-1}\right)\right\}\right) \geq 9 .
$$

This settles Case (2.2.4) and concludes Case (2.2).

Case (2.3): $\gamma\left(u_{1} u_{m-1}\right) \neq \gamma\left(u_{1} u_{3}\right), \gamma\left(u_{2} u_{m}\right)=\gamma\left(u_{m-2} u_{m}\right)$.

This case is symmetrical to Case (2.2). It follows from it by interchanging the variables $x, y$ and reversing the sequence $u_{1}, \ldots, u_{m}$.

Case (2.4): $\gamma\left(u_{1} u_{m-1}\right) \neq \gamma\left(u_{1} u_{3}\right), \gamma\left(u_{2} u_{m}\right) \neq \gamma\left(u_{m-2} u_{m}\right)$.

Being in Case (2), recall that

$$
A^{\prime}=A \sqcup\left\{u_{1} u_{m}\right\}=\left\{u_{1}^{2}, u_{1} u_{2}, \gamma\left(u_{1} u_{3}\right), \gamma\left(u_{m-2} u_{m}\right), u_{m-1} u_{m}, u_{m}^{2}, u_{1} u_{m}\right\},
$$

a subset of cardinality 7 in $\mathcal{G}\left(I^{2}\right)$. It follows from the present hypotheses that

$$
A^{\prime} \cap\left\{\gamma\left(u_{1} u_{m-1}\right), \gamma\left(u_{2} u_{m}\right)\right\}=\emptyset \text {. }
$$

Thus, it suffices to show $\gamma\left(u_{1} u_{m-1}\right) \neq \gamma\left(u_{2} u_{m}\right)$, for then this would imply

$$
\operatorname{card}\left(A^{\prime} \cup\left\{\gamma\left(u_{1} u_{m-1}\right), \gamma\left(u_{2} u_{m}\right)\right\}\right)=9
$$

and we would be done. Assume for a contradiction the contrary, i.e. that $\gamma\left(u_{1} u_{m-1}\right)=$ $\gamma\left(u_{2} u_{m}\right)$. Lemma 6.1 then implies

$$
\gamma\left(u_{1} u_{m-1}\right)=\gamma\left(u_{2} u_{m}\right)=u_{r} u_{s}
$$

for some $r, s \in[3, m-2]$ with $r \leq s$. Thus $u_{r} u_{s}$ divides both $u_{1} u_{m-1}$ and $u_{2} u_{m}$. Since $(1, m-1)<(1, m)<(2, m)$ in $V$, Lemma 2.3 implies that $u_{r} u_{s}$ also divides $u_{1} u_{m}$. But since $u_{1} u_{m} \in A^{\prime} \subseteq \mathcal{G}\left(I^{2}\right)$, it follows that $u_{r} u_{s}=u_{1} u_{m}$. Thus $u_{r} u_{s} \in A^{\prime}$, contrary to the conjunction of (17) and (15). This contradiction settles (16) and concludes the proof of the present last case, and hence of the theorem. 
For completeness, let us give optimal lower bounds on $\mu\left(I^{2}\right)$ for monomial ideals $I \subset K[x, y]$ such that $\mu(I)=m \leq 5$. The result is as follows.

$$
\mu\left(I^{2}\right) \geq \begin{cases}1 & \text { if } m=1 \\ 3 & \text { if } m=2, \\ 5 & \text { if } m=3, \\ 7 & \text { if } m=4 \\ 8 & \text { if } m=5\end{cases}
$$

As stated, these five lower bounds are sharp. The verification is left to the reader.

Let us conclude this paper with two questions. Let $m \geq 6$ be an integer, and let $I \subset K[x, y]$ be a monomial ideal such that $\mu(I)=m$. We have seen in Proposition 4.3 that if $I$ is generated in a single degree, then $\mu\left(I^{2}\right) \geq 2 m-1$. Our ideals in Proposition 4.1 reaching the absolute minimum $\mu\left(I^{2}\right)=9$ are generated in two degrees depending on $m$, namely $5 m$ and $7 m+3$.

Here is our first question. If $I$ is generated in two degrees $d_{1}<d_{2}$ such that the difference $d=d_{2}-d_{1}$ is fixed and independent of $m$, does it follow that $\mu\left(I^{2}\right)$ must grow to infinity with $m$ ? This seems to be true for $d=1$, but it would be nice to have a proof.

Our second question is, what would be the proper generalization of the present results in $n$ variables?

\section{REFERENCES}

[1] J. Herzog and R. Waldi, A note on the Hilbert function of a one-dimensional Cohen-Macaulay ring. Manuscripta Math. 16 (1975), 251-260.

[2] F. Orecchia, One-dimensional local rings with reduced associated graded ring and their Hilbert functions. Manuscripta Math. 32 (1980), 391-405.

[3] J. Herzog, M. Mohammadi Saem and N. Zamani, On the number of generators of powers of an ideal, 2017, arXiv:1707.07302.

Shalom Eliahou, Univ. Littoral Côte d'Opale, EA 2597 - LMPA - Laboratoire de Mathématiques Pures et Appliquées Joseph Liouville, F-62228 Calais, France and CNRS, FR 2956, FRANCE

E-mail address: eliahou@univ-littoral.fr

Jürgen Herzog, Fachbereich Mathematik, Universität Duisburg-Essen, Campus Essen, 45117 Essen, Germany

E-mail address: juergen.herzog@uni-essen.de

Maryam Mohammadi Saem, Faculty of Science, University of Mohaghegh Ardabili, P.O. Box 179, Ardabil, Iran

E-mail address: m.mohammadisaem@yahoo.com 